\title{
Some comments on the nonlinear dynamics of a portal frame under base excitation
}

\author{
Aline Souza de Paula ${ }^{\mathrm{a}, *}$, José Manoel Balthazar ${ }^{\mathrm{b}}$ and Jorge Luis Palacios Felix ${ }^{\mathrm{c}}$ \\ ${ }^{a}$ Department of Mechanical Engineering, Universidade de Brasília, Brasília, DF, Brazil \\ ${ }^{\mathrm{b}}$ Departamento de Estatística, Matemática Aplicada e Computação-UNESP, Instituto de Geociências e Ciências \\ Exatas, Universidade Estadual Paulista, Rio Claro, SP, Brasil \\ ${ }^{\mathrm{c}}$ Depto de Engenharias, Universidade Federal do Pampa, Campus Alegrete, RS, Brasil
}

\begin{abstract}
This paper presents a nonlinear dynamic analysis of a flexible portal frame subjected to support excitation, which is provided by an electro-dynamical shaker. The main goal of this study is to investigate the dynamic interactions between a flexible portal frame and a nonlinear electrical support excitation. The numerical analysis shows a complex behavior of the system, which can be observed by phase spaces, Poincaré sections and bifurcation diagrams.
\end{abstract}

Keywords: Electro-dynamical shaker, flexible portal frame, nonlinear dynamics

\section{Introduction}

Portal frames are commonly used in buildings and other structural configurations. The nonlinear vibrations of these systems have been investigated by several authors [1-3]. Brasil [4] presented the mathematical modeling of a portal frame excited by two ideal energy sources, consisting of unbalanced rotating machines, and performed an analytical study of its nonlinear vibrations. This same problem with two non-ideal sources was studied by Balthazar et al. [5], which focused on the self-synchronization of the energy sources when excitation was provided due to unbalanced rotating machines. When the excitation is not influenced by system response, it is called an ideal excitation or an ideal energy source. In contrast, when the excitation is influenced by the response of the system, it is known as non-ideal. Different authors studied dynamical system with non-ideal excitation [6-8].

Besides excitation due to unbalanced rotating machines, several authors analyze vibrating systems subjected to base excitation. Bar and McWannel [1] analyzed a frame under support motion considering linear elastic forces. Xu et al. [9] and De Paula et al. [10] performed numerical and experimental dynamical analysis of a parametrically excited pendulum. Jiang et al. [11] studied a tuning fork vibratory micromachined gyroscope subjected to base excitation.

The aim of this paper is to extend the above studies to an electromechanical device with flexible portal frame. This is a new interesting area of applications since many industrial tasks are carried out through flexible structures. The device under consideration here consists of a Duffing electrical circuit coupled magnetically to a flexible portal frame. This system modeling was previously addressed by Brasil [4] and Balthazar et al. [5].

In this paper it is carried out a dynamical analysis of a flexible portal frame under base excitation, which is provided by an electro-mechanical shaker. The electro-mechanical device considered in this work consists of an electric system magnetically coupled to a mechanical structure as used by Felix and Balthazar [12,13] and Yamapi [14] in others vibrating problems. De Paula et al. [15] presented preliminary results of the same problem studied in the present work.

\footnotetext{
*Corresponding author: Aline Souza de Paula, Department of Mechanical Engineering, Universidade de Brasília, 70.910.900, Brasília, DF, Brazil. E-mail: alinedepaula@unb.br.
} 


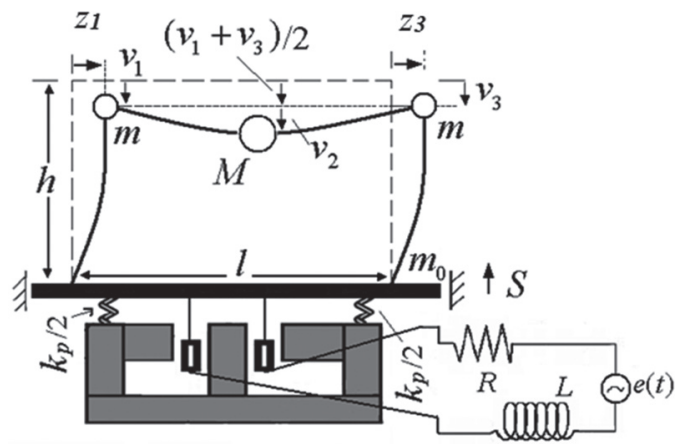

Fig. 1. Flexible portal frame and electro-dynamical shaker physical model [15].

\section{Flexible portal frame modelling}

The proposed system consists of a flexible portal frame (FPF) with base excitation provided by an electrodynamical shaker, as shown in the schematic picture presented in Fig. 1, at deflected position. The system consists of two columns connected by a beam at upper end and clamped in a base at lower end. The base is a platform of mass $m_{0}$ and stiffness $k_{p}$, with vertical displacement denoted by $S$. Each column has height $h$, flexural rigidity of $E I$ and carries a lumped mass $m$ at the upper end. The horizontal beam has length $l$, carries a central mass $M$ and is pinned to the columns at both ends.

The three concentrated masses of the flexible portal frame absolute coordinates can be written as:

$$
\begin{aligned}
& x_{1}=z_{1}, \quad x_{2}=\frac{l}{2}+z_{2}, \quad x_{3}=l+z_{3} \\
& y_{1}=h-v_{1}+S, \quad y_{2}=h+S-\frac{v_{1}+v_{3}}{2}-v_{2}, \quad y_{3}=h+S-v_{3}
\end{aligned}
$$

The linear stiffness of the columns and the beam can be evaluated by a Raleigh-Ritz procedure from cubic trial functions. Moreover, geometric nonlinearities are introduced by considering the shortening due to columns and beams bending under inextensibility hypothesis as presented by Brasil [4] and shown in Eq. (2).

$$
v_{1}=\frac{1}{2} A z_{1}^{2}, \quad v_{3}=\frac{1}{2} A z_{3}^{2}, \quad z_{2}=z_{1}-\frac{1}{4} B v_{2}^{2}, \quad z_{3}=z_{1}-\frac{1}{2} B z_{2}^{2}
$$

where the following constants are also obtained from cubic trial functions:

$$
A=\frac{6}{5 h}, \quad B=\frac{24}{5 l}
$$

Using algebraic relations presented in Eqs (1) and (2), the modeling of the flexible portal frame system may be reduced to two unknown variables: $z_{1}$ (horizontal displacement) and $v_{2}$ (vertical displacement).

The coupling between the flexible portal frame and the electro-dynamical shaker is then obtained through the electromagnetic force as presented in Eq. (4), caused by a permanent magnet.

$$
F_{e m}=K \dot{q}
$$

where $K=l_{c} B_{c}$, and $l_{c}$ and $B_{c}$ are, respectively, length of the conductor and magnetic flied. As a result, it produces a Laplace force in the mechanical part and the Lenz electromotive voltage in the electrical part. The electrical part of the system consists of a resistor $(R)$, an inductor $(L)$, a capacitor $(C)$ and a sinusoidal voltage source $\left(e(t)=e_{0} \cos (\omega t)\right)$ connected in series. In the present model, the capacitor voltage is a non-linear function of the instantaneous electrical charge $q$ as presented in Eq. (5).

$$
V_{c}=\frac{1}{C_{0}} q+d_{3} q^{3}
$$



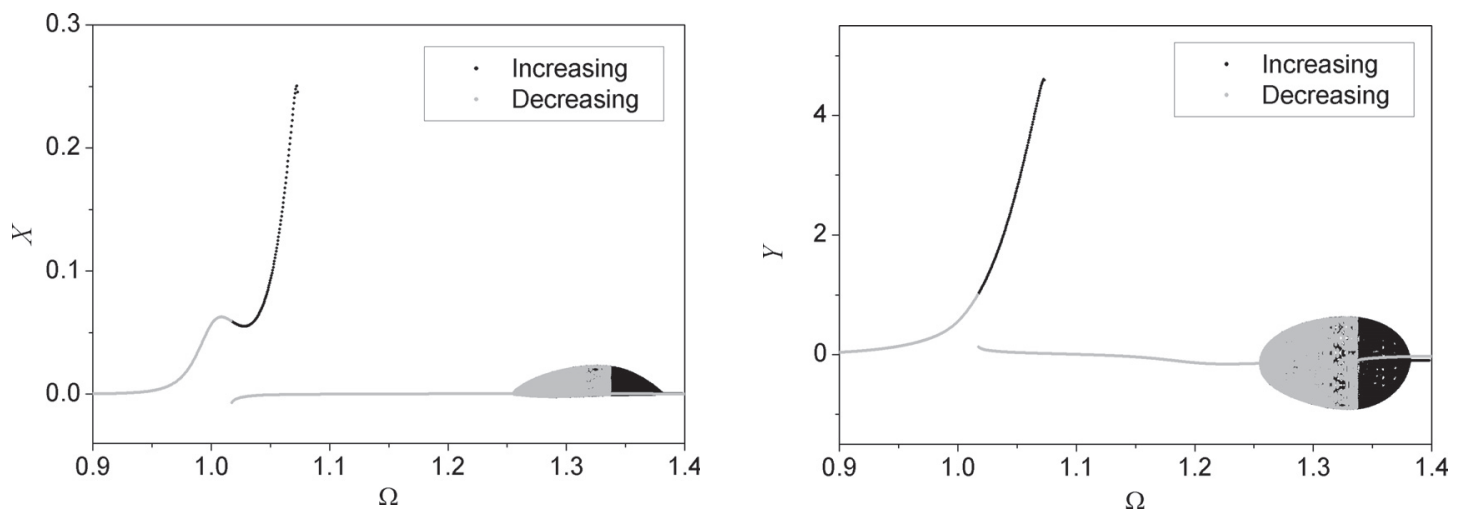

Fig. 2. Bifurcation diagram constructed by increasing and decreasing the forcing frequencies.

where $C_{0}$ is the linear part of $C$ and $d_{3}$ is the nonlinear coefficient, both depending on the type of the capacitor in use.

The system equations of motion can then be obtained by using the Lagrangian equations and are presented in Eq. (6) in dimensionless form. The portal frame governing equations without excitation are similar to those obtained by Balthazar et al. [5] and the coupling with the electro-dynamical shaker is obtained according to Felix and Balthazar [12].

$$
\begin{aligned}
& X^{\prime \prime}+\mu_{1} X^{\prime}+X=\alpha_{1}\left(Y^{\prime \prime} Y+Y^{2}\right)+\frac{\alpha_{1}}{2} Y^{2}+\frac{\alpha_{1}}{2} U^{\prime \prime} X-\delta_{1} X Y^{\prime \prime} \\
& Y^{\prime \prime}+\mu_{2} Y^{\prime}+w_{2}^{2} Y=\delta_{2} X^{\prime \prime} Y+\gamma_{2} X Y-g_{2}-\alpha_{2}\left(X^{\prime 2}+X X^{\prime \prime}\right)+\delta_{3} U^{\prime \prime} \\
& U^{\prime \prime}+\mu_{3} U^{\prime}+w_{3}^{2} U-\beta_{1} V^{\prime}=\alpha_{3}\left(X^{\prime 2}+X X^{\prime \prime}\right)+\delta_{4} Y^{\prime \prime}-g_{1} \\
& V^{\prime \prime}+\mu_{4} V^{\prime}+w_{4}^{2} V+\lambda_{3} V^{3}+\beta_{2} U^{\prime}=E_{0} \cos \Omega \tau
\end{aligned}
$$

where $\tau=\omega_{1} t, X=\frac{z_{1}}{h}, Y=\frac{v_{2}}{l}, U=\frac{S}{l}, V=\frac{q}{q_{0}}, \omega_{1}=\sqrt{\frac{2 k_{c}}{M+2 m}-A g}, \omega_{2}=\sqrt{\frac{k_{b}}{M+m}}, \omega_{3}=\sqrt{\frac{k_{p}}{M+2 m+m_{0}}}$,

$w_{2}=\frac{\omega_{2}}{\omega_{1}}, w_{3}=\frac{\omega_{3}}{\omega_{1}}, w_{4}=\frac{\omega_{e}}{\omega_{1}}, \Omega=\frac{\omega}{\omega_{1}}, \alpha_{1}=\frac{12 l}{5 h}, \delta_{1}=\frac{\alpha_{1} M}{2(M+2 m)}, \mu_{1}=\frac{c_{1}}{(M+2 m) \omega_{1}}, \delta_{2}=w_{2}^{2} \gamma_{2}, \gamma_{2}=\frac{12 h(M+2 m)}{5 l(M+m)}$,

$\mu_{2}=\frac{c_{2}}{(M+m) \omega_{1}}, g_{1}=\frac{g}{\omega_{1}^{2} l}, g_{2}=\delta_{3} g_{1}, \alpha_{2}=\frac{6 h \delta_{3}}{5 l}, \delta_{3}=\frac{M}{M+m}, \mu_{3}=\frac{c_{3}}{M_{t} \omega_{1}}, \beta_{1}=\frac{\mathrm{K} q_{0}}{M_{t} \omega_{1} l}, \alpha_{3}=\frac{6 h(M+2 m)}{5 l M_{t}}$,

$\delta_{4}=\frac{M}{M_{t}}, \mu_{4}=\frac{R}{L \omega_{1}}, \lambda_{3}=\frac{d_{3} q_{0}^{2}}{L \omega_{1}^{2}}, \beta_{2}=\frac{\mathrm{K} l}{L \omega_{1} q_{0}}, E_{0}=\frac{e_{0}}{L \omega_{1}^{2} q_{0}}, \Omega=\frac{\omega}{\omega_{1}}$ and $M_{t}=M+2 m+m_{0}$.

\section{Numerical results}

The dynamical analysis of the proposed system is carried out numerically by using the forth order Runge-Kutta method and appropriate tools for nonlinear dynamics investigation. An important characteristic associated with dynamical response of nonlinear system near resonance is the jump phenomena [16,17]. Jump phenomenon results in abrupt changes in system behavior and its analysis is essential for a proper design of nonlinear systems. Besides abrupt changes in system response, these jumps lead to system instability due to the coexistence of periodic attractors.

In this context, Fig. 2 shows bifurcation diagram with $E_{0}=0.15$ by increasing and by decreasing the forcing frequency and considering the following parameters: $\mu_{1}=0.05, \mu_{2}=0.05, \mu_{3}=0.01, \mu_{4}=0.01, \delta_{1}=0.05, \delta_{2}=$ $0.5, \delta_{3}=0.8, \delta_{4}=0.2, w_{2}=0.5, w_{3}=1, w_{4}=1, \alpha_{1}=0.05, \alpha_{2}=0.1, \alpha_{3}=0.4, g_{1}=0.001, g_{2}=0.0008, \beta_{1}=$ $0.4, \beta_{2}=0.2, \lambda_{3}=0.95, \gamma_{2}=0.05$ [12]. It can be observed the presence of jump phenomenon and also different behaviors of the system when increasing and decreasing the frequency. This different behaviors cause instability, since the system can present two different stable orbits for the same parameters depending on the initial condition. 


$$
0
$$



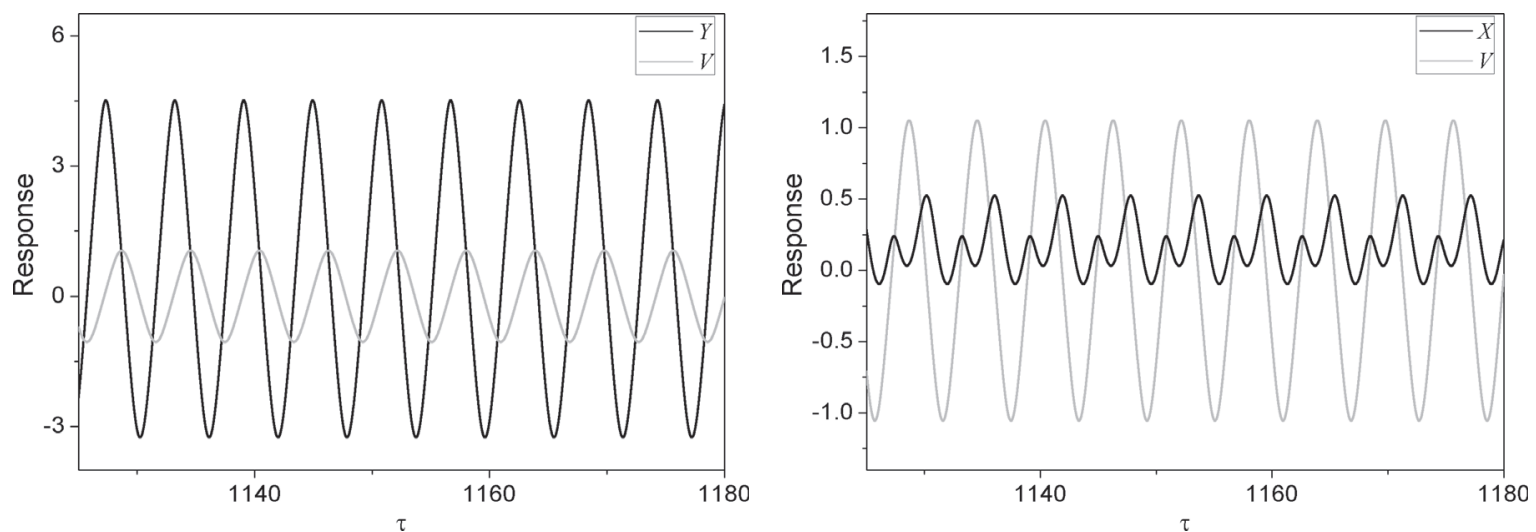

(a)
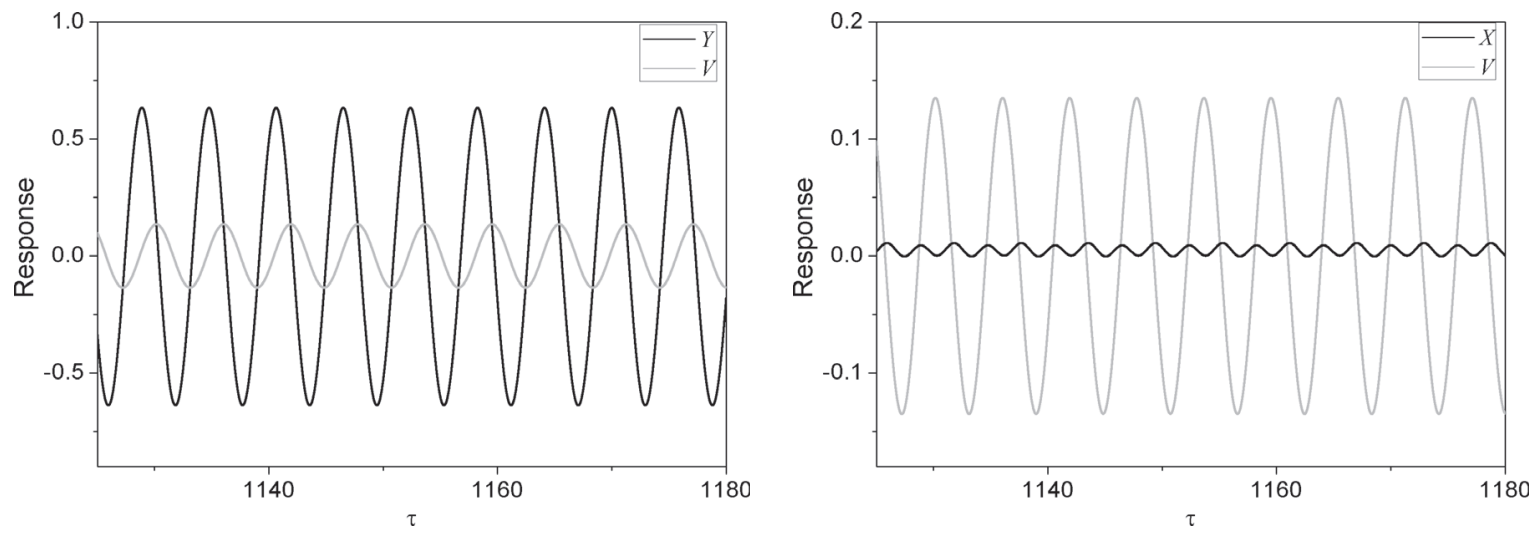

(b)

Fig. 4. Dynamical interaction between shaker and portal frame with $E_{0}=0.15$ and $\Omega=1.07$ (a) increasing and (b) decreasing the forcing frequency.

The bifurcation is constructed by assuming a quasi-static stroboscopically increase and decrease of the forcing frequency, $\Omega$, with $E_{0}=0.15$. Moreover, the first 1.000 forcing periods at each forcing frequency are neglected in order to reach the steady state response.

Besides jump phenomenon, the bifurcation diagram indicates a region related to quasi-periodic behavior as well as a region of coexistence of quasi-periodic and periodic attractors.

Figure 3(a) presents phase space of the coexistent orbits observed in Fig. 2 at $\Omega=1.07$. The black curve is related to system behaviour obtained when increasing the forcing frequency, while the green curve corresponds to system response obtained when decreasing the frequency. It can be observed a significant difference between the two stable orbits that coexists at the same system and forcing parameters. The change from one orbit to the other can occurs with slight external perturbations. These abrupt changes are dangerous to the dynamical system.

Figure 3(b) presents two coexisting attractors in phase space also observed in Fig. 2 now at $\Omega=1.35$. The quasi-periodic response is obtained when increasing the forcing frequency, represented by the black curve, while the periodic response is obtained when decreasing the frequency and is represented by the green curve.

Figures 4(a) and (b) shows electrical charge and flexible portal frame vertical displacement in time so that the dynamics interaction between the shaker and the flexible portal frame in steady state can be observed. Figure 4(a) shows the behavior obtained when increasing the forcing frequency, while Fig. 4(b) presents the response when decreasing the frequency.

Figure 5(a) presents phase space and Poincare section at $X-X^{\prime}, Y-Y^{\prime}$ and $V-V^{\prime}$ planes related to periodic orbit presented in Fig. 3(b), while Fig. 5(b) presents the same results for the quasi-periodic motion shown in Fig. 3(b).

Similarly to Fig. 4, Fig. 6 shows electrical charge and flexible portal frame vertical and horizontal displacements in time when increasing the forcing frequency. The dynamical interaction between shaker and flexible portal frame 

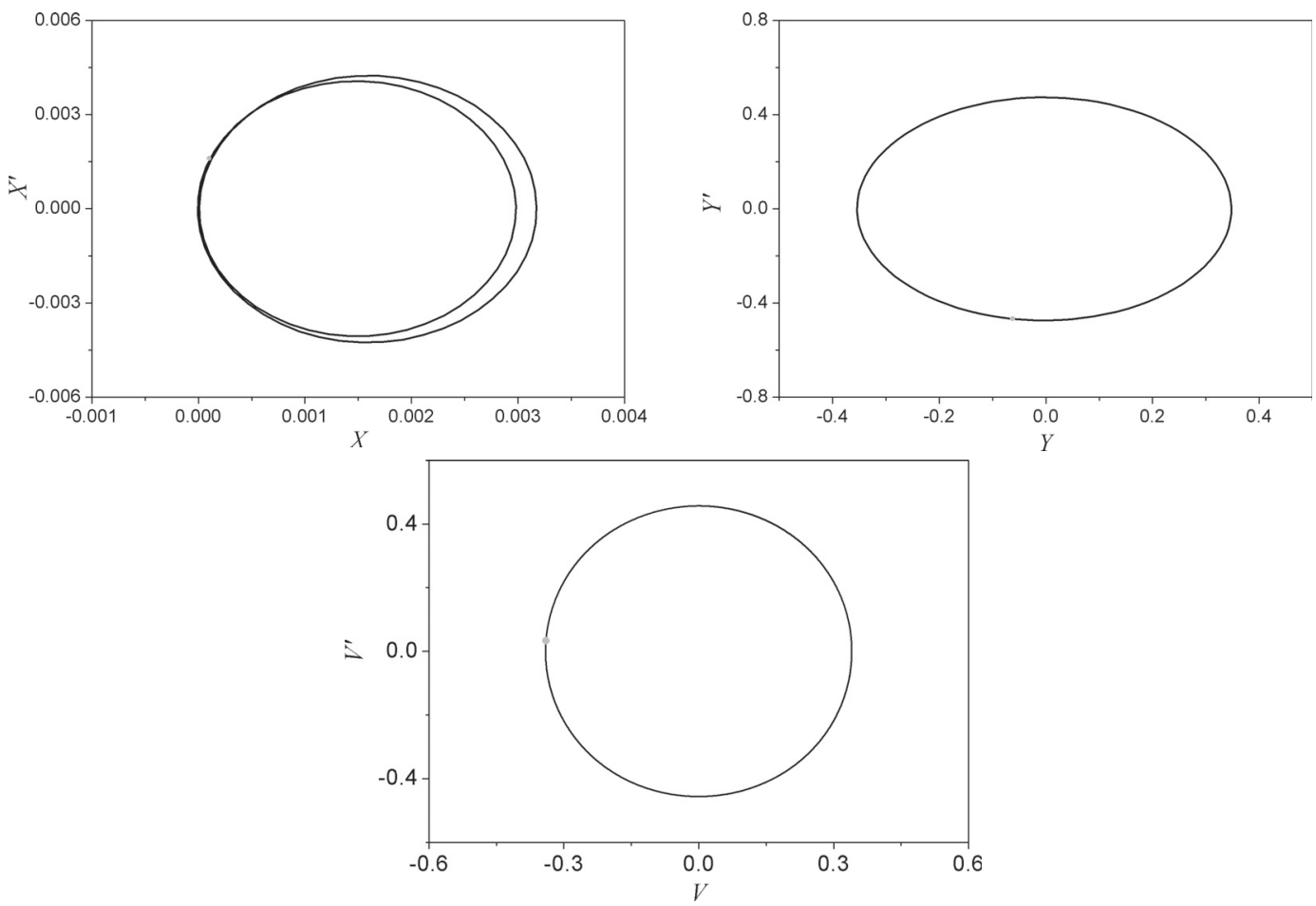

(a)
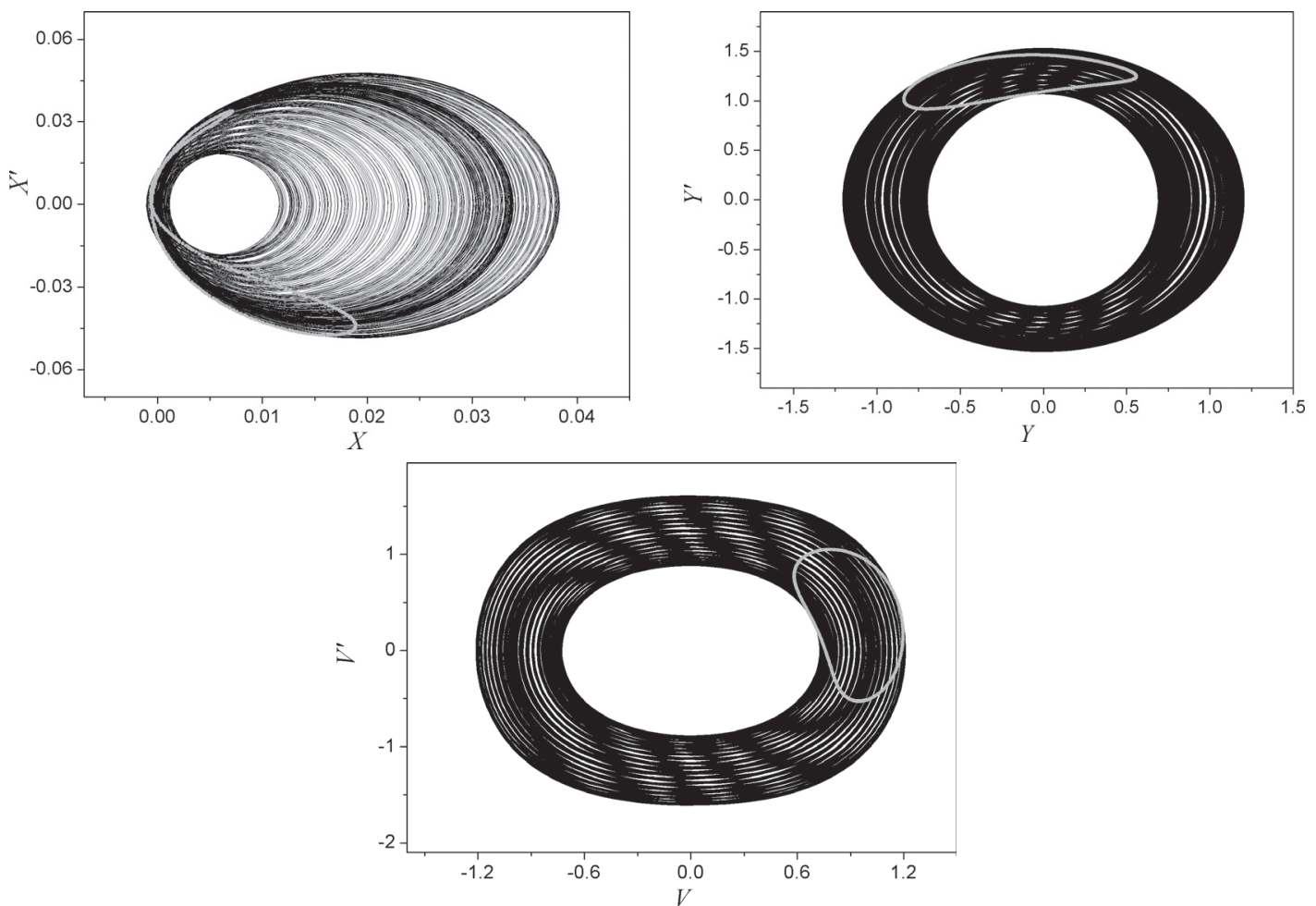

(b)

Fig. 5. Phase space and Poincare with $E_{0}=0.15$ and $\Omega=1.35$. Behavior obtained when (a) decreasing and (b) increasing the forcing frequency. 

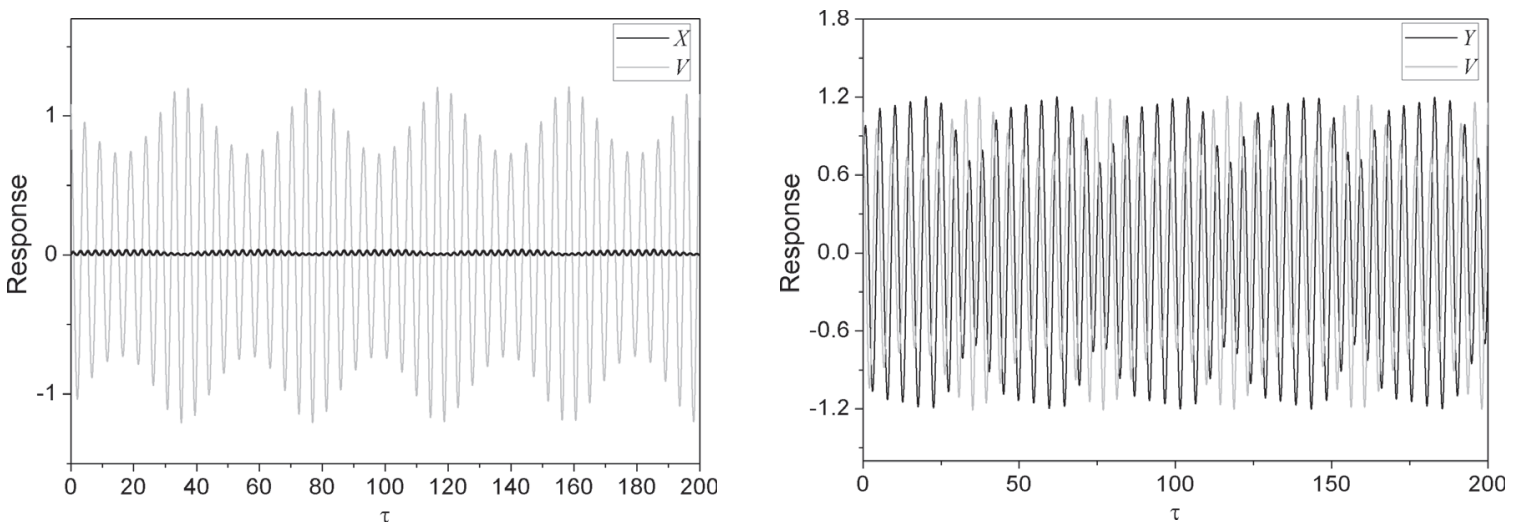

Fig. 6. Dynamical interaction between shaker and portal frame with $E_{0}=0.15$ and $\Omega=1.35$. Quasi-periodic behavior.

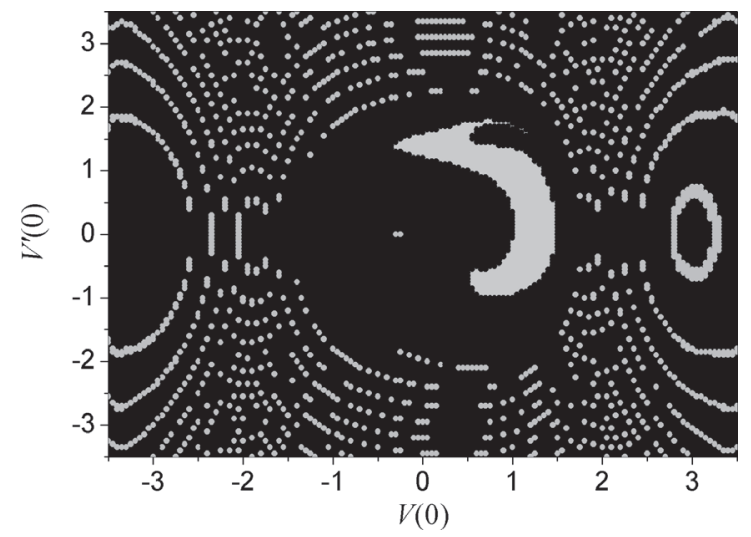

Fig. 7. Basin of attraction with $E_{0}=0.15, \Omega=1.35,\left\{X(0), X^{\prime}(0), Y(0), Y^{\prime}(0), U(0), U^{\prime}(0)\right\}=\{0,0,1,0,0,0\}$.

can be observed, when the shaker electrical charge increases, the vertical displacement decreases and vice-versa.

The coexisting attractors identified with $E_{0}=0.15$ and $\Omega=1.35$ are now analyzed by constructing the basin of attraction. Figure 7 presents the basin of attraction for different initial conditions of $V$ and $V^{\prime},\left\{X(0), X^{\prime}(0), Y(0)\right.$, $\left.Y^{\prime}(0), U(0), U^{\prime}(0)\right\}=\{0,0,1,0,0,0\}$ and initial forcing phase $\theta_{F}(0)=\Omega \tau(0)=0$. Three coexisting behaviors are observed. Green points correspond to the period-1 orbit shown in Fig. 5(a), gray points correspond the quasiperiodic response shown in Fig. 5(b), while the black points correspond to another quasi-periodic response. This second quasi-periodic attractor is presented in Fig. 8 in phase space and Poincaré section. All responses identified in the basin of attraction correspond to steady state response.

At this point different initial values for $Y(0)$ are considered to construct the basin of attraction. All other parameters and initial conditions are equal to the ones associated with the basin of attraction shown in Fig. 7. The identified behaviors, and the associated colors, are the same as presented before. Figure 9 presents the basin of attraction for different initial conditions for $V$ and $V^{\prime},\left\{X(0), X^{\prime}(0), Y(0), Y^{\prime}(0), U(0), U^{\prime}(0)\right\}=\{0,0,0,0,0,0\}$ and initial forcing phase $\theta_{F}(0)=\Omega \tau(0)=0$. For these initial conditions the first quasi-periodic behavior, presented in Fig. 5(b), is not observed.

Figure 10 presents the basin of attraction for different initial conditions for $V$ and $V^{\prime},\left\{X(0), X^{\prime}(0), Y(0), Y^{\prime}(0)\right.$, $\left.U(0), U^{\prime}(0)\right\}=\{0,0,1.5,0,0,0\}$ and initial forcing phase $\theta_{F}(0)=\Omega \tau(0)=0$. For these initial conditions the first quasi-periodic behavior, presented in Fig. 5(b), is observed again. From various basin of attractions constructed, it was observed that presence of the first quasi-periodic behavior depends mainly of initial conditions of $Y, Y^{\prime}, V$ and $V^{\prime}$.

In each basin of attraction the initial conditions of two state variables are varied and all other initial conditions are fixed. Thus, since the system has six state variables, different basins of attractions must be considered to obtain all 

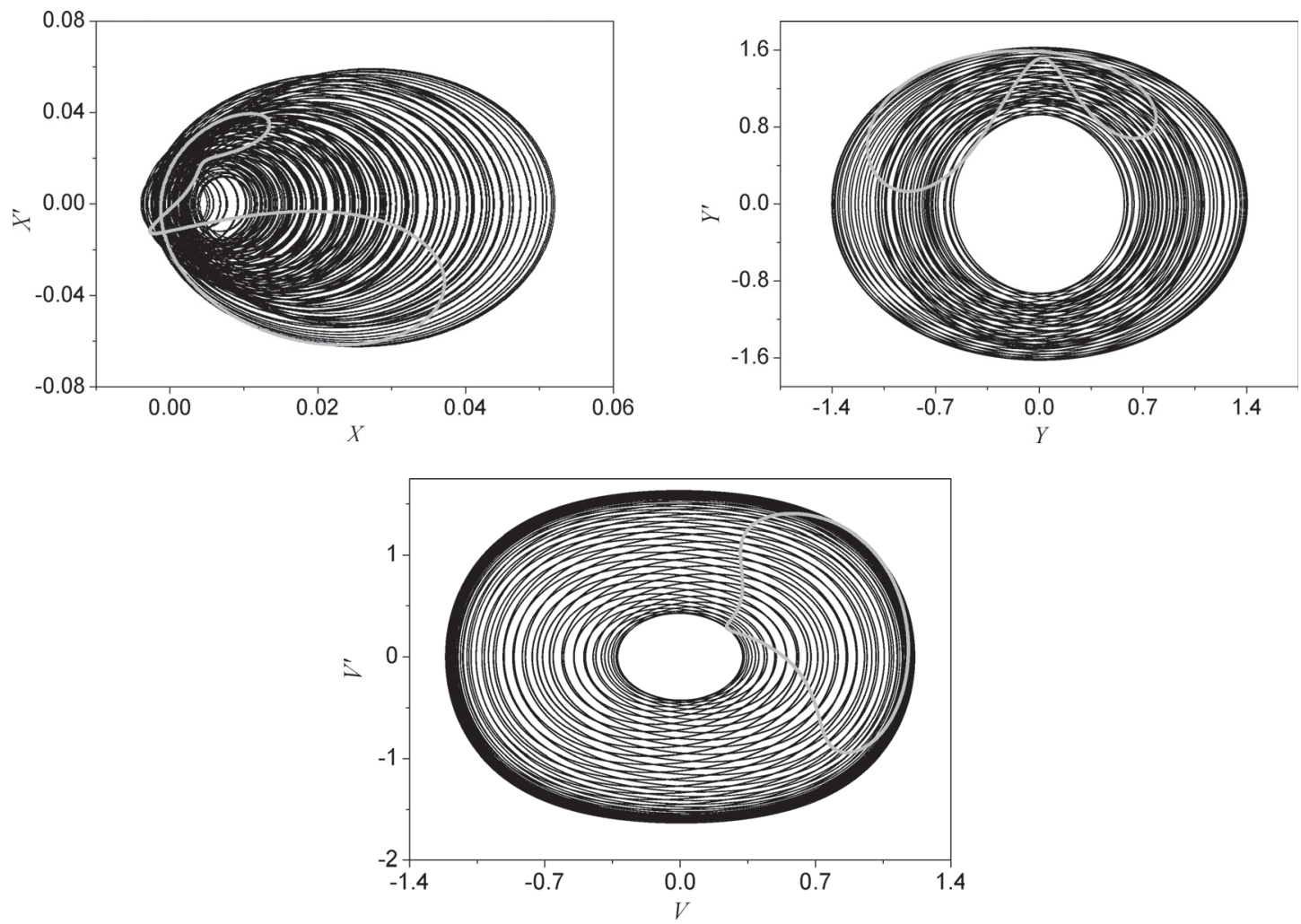

Fig. 8. Poincaré section of the quasi-periodic attractor with $E_{0}=0.15$ and $\Omega=1.35\left(\theta_{F}(0)=0\right)$.

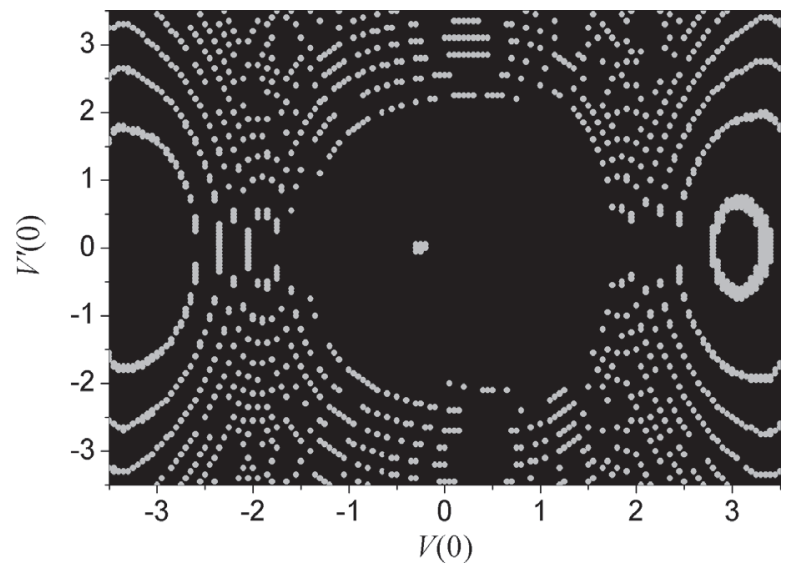

Fig. 9. Basin of attraction with $E_{0}=0.15, \Omega=1.35,\left\{X(0), X^{\prime}(0)\right.$, $\left.Y(0), Y^{\prime}(0), U(0), U^{\prime}(0)\right\}=\{0,0,0,0,0,0\}$.

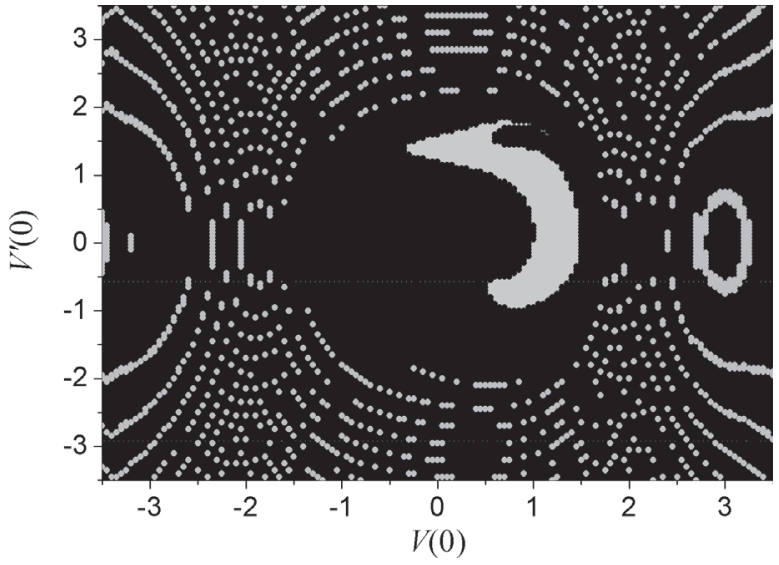

Fig. 10. Basin of attraction with $E_{0}=0.15, \Omega=1.35,\{X(0)$, $\left.X^{\prime}(0), Y(0), Y^{\prime}(0), U(0), U^{\prime}(0)\right\}=\{0,0,1.5,0,0,0\}$.

coexisting stable responses. From various constructed basins of attractions, it was observed the coexistence of two or three attractors as illustrated in Figs 7-10. The analysis of basin of attraction is very important to have a good knowledge of the system and avoid undesired behavior, such as abrupt changes in system dynamics, which can be catastrophic. This study can be also very useful for control proposes. 


\section{Conclusions}

In this work a flexible portal frame parametrically excited by an electro-dynamical shaker is considered. The dynamical analysis of the system is carried out numerically considering different forcing frequencies, mainly near the resonance region. The occurrence of jump phenomenon is verified, which is an important characteristic of nonlinear dynamical systems. Basically, jump phenomenon are associated with nonlinear resonant response, causing abrupt changes in system behavior and introducing unstable regions on system response. The unstable region is caused by the presence of coexisting periodic attractors, as presented in the results. Thus, jump phenomenon and coexistence of stable attractors are important characteristics that must be analyzed to provide good system knowledge and avoid undesired behavior. In this paper the coexistence of stable solutions were analyzed by basin of attractions which showed the coexistence of two and even three attractors. Besides the jump phenomenon, an important point associated with the proposed system is the dynamical interaction between the shaker and the FPF, especially when system presents quasi-periodic response.

\section{Acknowledgments}

The authors would like to thank the Brazilian Research Agencies CNPq and CAPES, and through the INCT-EIE (National Institute of Science and Technology - Smart Structures in Engineering) the CNPq and FAPEMIG for their support.

\section{References}

[1] A.D.S. Barr and D.C. McWannell, Parametric instability in structures under support motion, Journal of Sound and Vibration 14(4) (1971), 491-509.

[2] G.J. Simitse, J.D. Swisshelm and A.S. Vlahinos, Flexibly-jointed unbraced portal frames, J Construct Steel Research 4 (1984), $27-44$.

[3] C.E.N. Mazzilli and R.M.L.R.F. Brasil, Effect of static loading on the nonlinear vibrations of a three-time redundant portal frame: Analytical and numerical studies, Nonlinear Dynamics 8 (1995), 347-366.

[4] R.M.L.R.F. Brasil, Multiple scales analysis of nonlinear oscillations of a portal frame fondations for several machines, Journal of the Brazilian Society of Mechanical Sciences 21(4) (1999), 641-654.

[5] J.M. Balthazar, J.L.P. Felix and R.M.L.R.F. Brasil, Short comments on self-synchronization of the non-ideal sources supported by a felxible portal frame structure, Journal of Vibration and Control 10 (2004), 1739-1748.

[6] A.H. Nayfeh and D.T. Mook, Nonlinear Oscillations, Wiley and Sons, 1979.

[7] J.M. Balthazar, D.T.Mook, H.I. Weber, R.M.L.R.F. Brasil, A. Fenili, D. Belato and J.L.P. Felix, An overview on non-ideal vibrations, Meccanica 38 (2003), 613-621.

[8] M.J.H. Dantas and J.M. Balthazar, On the existence and stability of periodic orbits in non-ideal problems: General results, ZAMM- Journal of Applied Mathematics and Mechanics 58 (2007), 940-956.

[9] X. Xu, E. Pavlovskaia, M. Wiercigroch, F. Romeo and S. Lenci, Dynamic interactions between parametric pendulum and electro-dynamical shaker, Z Angew Math Mech 87(2) (2007), 172-186.

[10] A.S. De Paula, M.A. Savi, M. Wiercigroch and E. Pavlovskaia, Bifurcation control of a parametric pendulum, International Journal of Bifurcation and Chaos in Applied Sciences and Engineering 22(1250111) (2012), 14.

[11] T. Jiang, A. Wang and G. Liu, Detection capacitance analysis method for tuning fork micromachined gyroscope based on elastic body model, Sensors and Actuators A 128 (2006), 52-59.

[12] J.L.P. Felix and J.M. Balthazar, Comments on a nonlinear and nonideal electromechanical damping vibration absorber, sommerfeld effect and energy transfer, Nonlinear Dynamics (2008), Doi: 10.2007/s11071-008-9340-8.

[13] J.L.P. Felix and J.M. Balthazar, On a non-ideal and nonlinear tuning fork micro-gyroscope dynamics, in: Twelfth Conference on Nonlinear Vibrations, Dynamics, and Multibody Systems (1-5 Jun 2008), VT, Blacksburg, VA, USA.

[14] R. Yamapi, Dynamics of an electromechanical damping device with magnetic coupling, Communication in Nonlinear Science and Numerical Simulation 11 (2006), 907-921.

[15] A.S. De Paula, J.M. Balthazar and J.L.P. Felix, Nonlinear dynamics of a flexible portal frame under support excitation, in: Procedings of the ICNPAA - World Congress: 9th International Conference on Mathematical Problems in Engineering, Aerospace and Science, Vienna, Austria (Jul 2012) American Institute of Physics.

[16] D. Bernardini and G. Rega, Thermomechanical modelling, nonlinear dynamics and chaos in shape memory oscillators, Mathematical and Computer Modelling of Dynamical Systems 11(3) (2005), 291-314.

[17] M.A. Savi, A.S. De Paula and D. Lagoudas, Numerical investigation of an adaptive vibration absorber using shape memory alloys, Journal of Intelligent Material Systems and Structures 22 (2011), 67-80. 

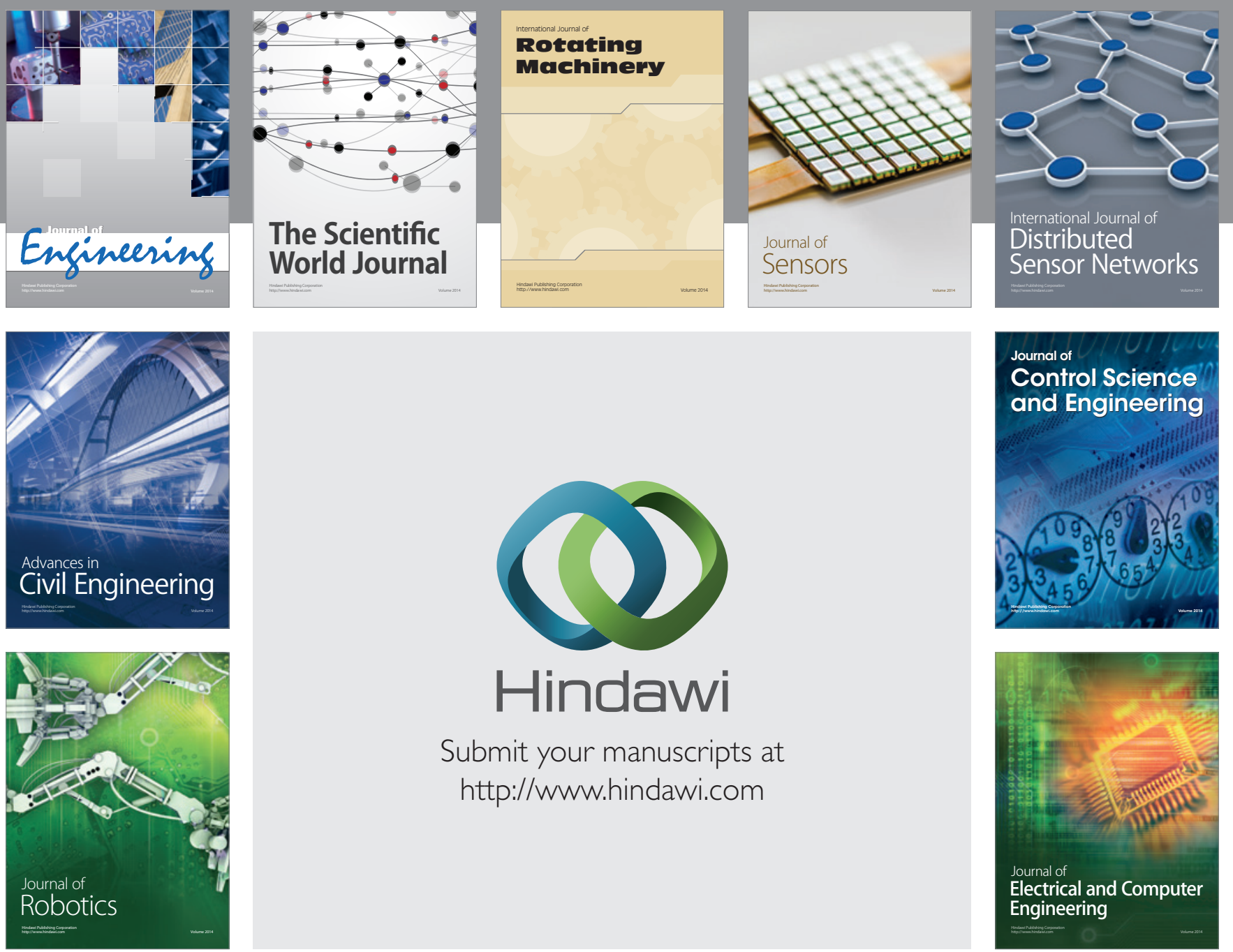

Submit your manuscripts at

http://www.hindawi.com
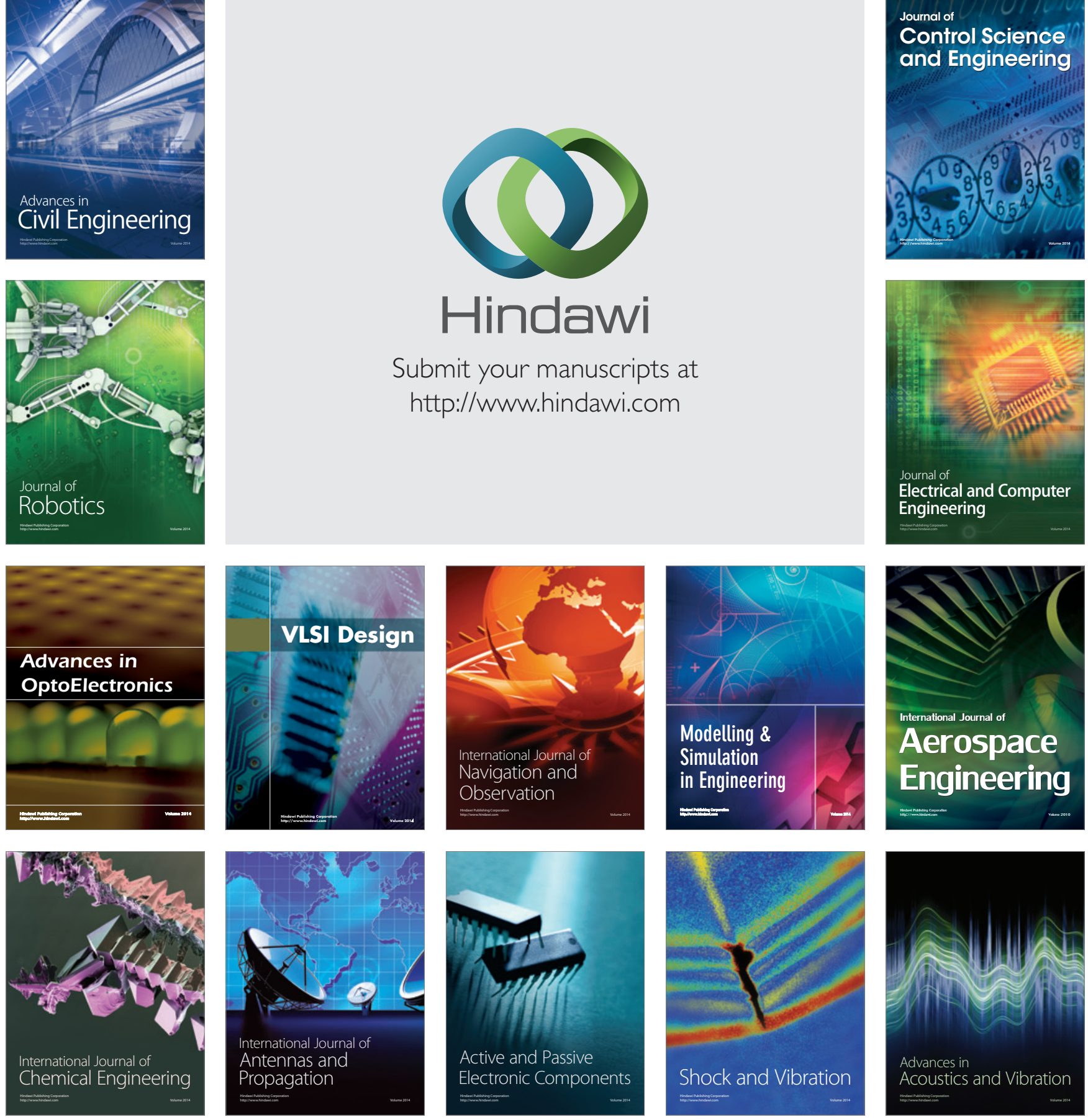